Literatura y Lingüística $\mathrm{N}^{\circ} 24$

ISSN 0716-5811 / pp. 143-159

\title{
Escritura académica: un modelo metodológico efectivo basado en tareas y enfoque cooperativo*
}

\author{
Angie Neira Martínez **, Anita Ferreira Cabrera***
}

\section{Resumen}

La importancia de la alfabetización académica ha sido reconocida por las instituciones de educación superior, así como la necesidad de desarrollarla a través de metodologías eficientes. En concordancia con aquello, esta investigación tiene como objetivo proponer un modelo metodológico para que estudiantes universitarios sean capaces de producir informes académicos estructural y lingüísticamente adecuados. Para ello, se ha probado empíricamente su efectividad a través de un estudio experimental longitudinal con pre y post test, y grupo control, y los resultados demuestran que, por medio de tareas y de la cooperación, es posible el aprendizaje de una estructura textual compleja y convencional, además de entender su función social.

Palabras clave: informe académico, escritura, enseñanza de lenguas, aprendizaje por tareas, aprendizaje cooperativo

\section{Academic writing: an effective methodological model based on tasks and cooperative learning}

\begin{abstract}
The relevance of academic literacy and its development through efficient methodologies have been recognized and considered an important need by higher education institutions. Bearing this in mind, the purpose of this study is to propose a methodological model that enables university students to produce structurally and linguistically adequate academic reports. In order to validate its effectiveness, such methodology was tested in an experimental pre-test/post-test control group longitudinal research design. The findings suggest that the learning of a complex and conventional textual structure, as well as its social function, is possible by adopting tasks in a cooperative environment.
\end{abstract}

Key words: academic report, writing, language teaching, task based learning, cooperative learning

Recibido: 25-03-2011

Aceptado: 05-04-2011

* Tesis para optar al grado de Magíster en Lingüística.

** Magíster en Lingüística. Estudiante de Doctorado en Lingüística. Becaria CONICYT. Universidad de Concepción. angieneira@udec.cl

*** Doctora en Lingüística. Doctora en Inteligencia Artificial. Universidad de Concepción. aferreir@udec.cl 
Escritura académica: un modelo metodológico efectivo basado en tareas y enfoque cooperativo / Angie Neira Martínez y Anita Ferreira Cabrera

\section{Introducción}

La escritura es la principal forma de interacción en las comunidades discursivas especializadas, es de esta manera como se publica, valida y transmite el conocimiento. Para que un estudiante que ingresa a la universidad pueda integrarse con éxito a la comunidad disciplinar en la que se enmarca su carrera, es fundamental que desarrolle sus habilidades lingüísticas en el ámbito académico, en especial, la producción y comprensión de textos escritos.

La alfabetización académica busca proveer al estudiante herramientas para que sea capaz de producir textos, no sólo de redactarlos. Esto quiere decir que la producción de textos incorpora el conocimiento de los géneros discursivos académicos y sus características, el manejo del registro que debe usarse en cada uno, la reflexión en torno al proceso de escritura, además de la aplicación de mecanismos de cohesión.

Uno de los géneros a los que más se enfrenta un estudiante universitario es el informe académico. Este género es heterogéneo, pues existe una gran gama de informes dependiendo de su propósito y de la disciplina en la que se produzca. Es así, como existen informes de lectura, informes de laboratorio, informes de salida a terreno, informes de práctica, entre otros. Este trabajo se centra en el informe académico de investigación bibliográfica, un texto que exige la activación de procesos de lectura, selección de información y escritura.

La investigación que se expone a continuación presenta la aplicación de una metodología de enseñanza basada en tareas y en el aprendizaje cooperativo, cuyo fin es que los estudiantes conozcan y manejen la estructura del informe académico de investigación bibliográfica y mejoren sus habilidades de escritura.

\section{Marco Teórico}

\subsection{El Proceso de escritura}

Para escribir, el autor debe realizar distintas actividades de pensamiento superior, desde que se plantea la tarea en un contexto social determinado, hasta que termina su texto. En esta práctica influyen diversos factores que participan e interactúan en el desarrollo del discurso, los cuales han sido descritos desde diferentes puntos de vista que confluyen en una idea común: la escritura es un proceso complejo y recursivo. 
Esta idea ha guiado la conformación de un paradigma que aparece a comienzos de la década de 1980, que es el de la escritura como un proceso. Éste es definido por Marinkovich (2002: 226) como "no prescriptivo, centrado en el proceso, el que, a la luz de los aportes derivados de las teorías psicológicas, sociales y lingüísticas hoy legitimadas, permite acercarse a lo que realmente sucede cuando se aprende a escribir".

Los primeros modelos de este paradigma son el de Flower y Hayes (1981) y el de Bereiter y Scardamalia (1987). Éstos, con influencias de la psicología cognitiva, proponen dos teorías que aún perduran, y que sentaron el precedente de modelos posteriores.

El modelo de Flower y Hayes (1981) se centra en el escritor y en los procesos mentales que éste lleva a cabo durante la escritura. Éstos serían recursivos y no lineales. El principal aporte de este modelo es considerar a la composición escrita como un proceso jerárquico, centrado en el escritor y guiado por metas.

El principal aporte del modelo de Bereiter y Scardamalia (1987) es el de reconocer que existen diferencias, según el nivel de experiencia y competencia del individuo y la naturaleza de las tareas de escritura. A raíz de ello, proponen la existencia de dos modelos diferentes de escritura: "decir el conocimiento" y "transformar el conocimiento". El primero es un proceso natural y cotidiano, pero, a la vez, limitado. El segundo describe el desarrollo de la tarea de escritura como un proceso que requiere un uso de habilidades que va más allá de lo cotidiano, un re-procesamiento del conocimiento, un razonamiento formal y problemático.

Por su parte, Grabe y Kaplan (1996) proponen un modelo comunicativo del proceso de escritura, que incorpora componentes cognitivos, sociales y textuales. A través de este modelo buscan explicar, principalmente, la escritura académica. Los autores indican que el propósito de proponer este modelo es proveer una forma de integrar lo que ellos consideran las tres mayores consideraciones para una teoría de la escritura, que son: el procesamiento cognitivo del escritor, los recursos lingüísticos y textuales que participan de la tarea de escritura y los factores contextuales que fuertemente moldean la naturaleza de la escritura.

Además, Chenowith y Hayes (2001) proponen un modelo concebido en tres niveles de procesamiento, que tiene como núcleo el procesamiento cognitivo de la escritura, incluyendo componentes externos que inciden en ella. 
Escritura académica: un modelo metodológico efectivo basado en tareas y enfoque cooperativo / Angie Neira Martínez y Anita Ferreira Cabrera

Los cuatro modelos de escritura citados coinciden en la importancia que le atribuyen al escritor y a lo que incide en cómo éste lleva a cabo una tarea de escritura. Si bien se han ido agregando variables y modificando levemente algunas, la base de la escritura como proceso se mantiene como un paradigma en constante desarrollo.

\subsection{El género informe académico}

El informe académico es un género discursivo especializado de vital importancia en el desarrollo de los procesos estudiantiles universitarios. Éste se construye en una situación comunicativa determinada, en la cual un estudiante demuestra a un experto (profesor o evaluador) los conocimientos adquiridos acerca de un tema o procedimiento específico.

Figueras y Santiago (2000) indican que los textos académicos comparten convenciones de estilo y se estructuran a partir de las mismas secuencias textuales de base. El estilo se caracteriza por ser preciso, claro y objetivo, esto es, el escritor debe evitar las ambigüedades a través del uso de léxico especializado y de la lengua estándar, hacer uso planificado del lenguaje y omitir marcas de implicación personal en el texto.

Tapia, Burdiles y Arancibia (2003) caracterizan al informe académico como un texto principalmente referencial-representativo, que tiene como objetivo ser soporte y transmisor del conocimiento. Además, agregan, es un discurso en cuya elaboración se utiliza un registro formal, un lenguaje objetivo y un léxico específico. Para producir este tipo de texto, el estudiante debe llevar a cabo una serie de tareas "de un nivel cognoscitivo superior y que requieren de una interacción armónica entre las habilidades cognitivas y las habilidades comunicativas" (Tapia, Burdiles y Arancibia, 2003: 251)

En cuanto a su estructura, Botta (2002) afirma que el informe académico debe comenzar con una introducción, continuar con un cuerpo y finalizar con la presentación de conclusiones. En la introducción, se explicita el interés que el fenómeno descrito despierta y una delimitación de dicha descripción. En el cuerpo, se exponen de manera objetiva, clara y ordenada los fenómenos observados, con el uso, en muchos casos, de fuentes informativas. Además, el texto del cuerpo puede ir complementado por cuadros, organigramas o imágenes que faciliten su interpretación. Finalmente, se presentan las conclusiones obtenidas a partir de los materiales informativos expuestos y se señalan aquellos aspectos que debieran ser estudiados más ampliamente. 
Tapia, Burdiles y Arancibia (2003) afirman que la organización de la información en este género discursivo es de carácter jerárquico, de manera de que se despliegan conceptos e ideas en un formato que limita el proceso de composición. Además, hay en este tipo de texto un componente intertextual que subyace a la consulta de diversas fuentes para su elaboración.

A partir del estudio de Harvey y Muñoz $(2006)^{1}$, y de lo que los académicos mencionan en él, el informe académico debería tener la siguiente estructura: un texto tripartito, compuesto por una introducción, un desarrollo y una conclusión; sin errores de redacción, fiel a los formatos preestablecidos y que plantee un punto de vista del estudiante fundamentado en relación con el tema tratado. Variaría, sin embargo, de un área a otra en las formas de organización del discurso que predominan, ya que las ciencias humanas y sociales lo conciben como descriptivo, mientras las ciencias matemáticas y naturales, como explicativo y resolutivo.

\subsection{Enfoques Metodológicos}

\subsubsection{El enfoque por tareas}

Este enfoque metodológico se centra en el desarrollo de la competencia comunicativa del estudiante, ligado a la capacidad de éste de ser agente activo de su propio aprendizaje. En este enfoque, las actividades son las que articulan las unidades didácticas.

Existen varias definiciones de tarea y todas coinciden en que es ésta el componente central del aprendizaje. Según Nunan (1989), una tarea es un trabajo que lleva a los alumnos a la comprensión, producción e interacción de la lengua, sin explicitación de las formas, sino enfocado en el significado. Para Estaire (2004), la tarea es una unidad de organización del aprendizaje que ofrece un contexto apropiado para el desarrollo del conocimiento formal (saber cosas sobre la lengua) y el conocimiento instrumental (saber utilizar la lengua como instrumento de comunicación) de manera paralela e integrada. Skehan (2008) define tarea como una actividad en la cual el significado es lo primordial, en la que hay

1 Harvey y Muñoz (2006) aplicaron 120 cuestionarios a académicos de tres instituciones universitarias de la Región Metropolitana, de ocho carreras diferentes, pertenecientes a las distintas áreas como ingeniería, letras, medicina, etc.; y en los cuales las preguntas eran dirigidas a conocer lo que éstos entendían por informe académico y qué esperaban que sus alumnos escribieran en él. 
Escritura académica: un modelo metodológico efectivo basado en tareas y enfoque cooperativo / Angie Neira Martínez y Anita Ferreira Cabrera

que solucionar problemas de comunicación, la que tiene relación con las actividades de la vida real.

\subsubsection{Diseño de un curso basado en tareas}

Nunan (2001) indica que, para elaborar un syllabus basado en tareas, quien lo diseña debe analizar las necesidades de sus estudiantes en términos de desarrollar tareas que ellos tengan que enfrentar en el mundo real, como lo es, en el caso de esta investigación, el informe académico de investigación bibliográfica. Para el diseño del curso aplicado en esta investigación, se consideraron tres clasificaciones y/u organizaciones de tarea: la propuesta por Nunan (2001), la planteada por Estaire (2004) y la sugerida por Skehan (2008).

Nunan (2001) clasifica las tareas en dos grupos: tareas del mundo real o tareas objetivo (real - world or target task) y tareas pedagógicas (pedagogical tasks). Las primeras se refieren a un tipo de tarea comunicativa que se logra en el mundo, fuera de la sala de clases. Las segundas son aquellas en las que los estudiantes trabajan en el aula comprendiendo, produciendo o interactuando la lengua (o con la lengua), mientras su atención está principalmente puesta en el significado más que en la forma.

Estaire (2004), por su parte, propone una clasificación de tareas según su función dentro de un curso de lengua: de comunicación y de apoyo lingüístico. Las primeras son unidades de trabajo en el aula centradas en el significado, es decir, en el qué se dice más que en el cómo (no debe olvidarse que el significado y la forma están íntimamente ligados, por lo que no pueden ser disgregados del todo). Las segundas son unidades de trabajo que tienen como núcleo el desarrollo de los contenidos lingüísticos (gramática, vocabulario, discurso, etc.), por lo cual la atención de los estudiantes está en la forma de la comunicación, en los aspectos lingüísticos propiamente tales.

Estos dos tipos de tareas se encuentran enmarcados dentro de una unidad didáctica. Ésta está compuesta por una serie de horas de clase, tiene como objetivo el desarrollo a la par de los conocimientos instrumentales y los formales y permite, por medio de una estructuración coherente de tareas, evaluar el aprendizaje a lo largo del proceso.

Finalmente, Skehan (2008) sugiere una clasificación en tres etapas: las pre-tareas, las tareas de proceso y las post-tareas. A pesar de que la primera y la tercera fases no son obligatorias, pueden jugar un rol importante en la efectividad de la metodología. 
La etapa de pre-tareas (pre-task phase) tiene como objetivo preparar al estudiante para la realización de las tareas de proceso. Hay dos alternativas en esta fase: poner el énfasis en las demandas cognitivas o poner el énfasis en los factores lingüísticos.

La etapa de las tareas de proceso (during task phase) puede ser dividida en dos tipos: las opciones de desarrollo y las opciones de proceso. Dentro de las opciones de desarrollo se pueden diferenciar tres consideraciones: la primera, si se requerirá que los estudiantes realicen la tarea bajo presión de tiempo o no; la segunda, si se permitirá el acceso de los alumnos a información de input mientras la llevan a cabo; y la tercera, si se le introducirá algún elemento sorpresa. Las opciones de proceso se refieren a la forma en la que el discurso que surge desde la tarea es representado. Éstas aluden a las decisiones on line tanto del profesor, acerca de cómo conducir el discurso para que refleje el conocimiento instrumental del alumno; como del estudiante, que manifiesta sus creencias acerca del aprendizaje de lenguas y acerca de una tarea en específico.

La etapa de post-tareas (post task phase) presenta una gran cantidad de opciones que podrían cumplir con una de las tres metas pedagógicas propuestas por Ellis (2003): proveer una oportunidad para repetir el desarrollo de la tarea, promover la reflexión de cómo se desarrolló y fomentar la atención en la forma, en especial de aquellas formas problemáticas que se presentaron en su realización.

Finalmente, una metodología basada en tareas es una manera participativa de enseñar, ya que no se fundamenta en entregar contenidos expositivamente durante una clase, sino que permite la interacción y el descubrimiento del conocimiento por parte de los alumnos, lo que les deriva responsabilidad en su propio aprendizaje, dándoles mayor autonomía y posibilitando al docente ser un guía de aprendizajes más que una enciclopedia que sabe y transmite.

\subsubsection{El aprendizaje cooperativo}

Se llama aprendizaje cooperativo a aquella organización de la clase en pequeños grupos de trabajo. Sin embargo, no todos los trabajos realizados en grupo pueden considerarse de aprendizaje cooperativo. Para que éste ocurra deben tomarse en cuenta una serie de factores. Como indica Trujillo (2002), las competencias lingüístico-comunicativas no son las únicas que se desarrollan a través del aprendizaje cooperativo, sino también las competencias sociales y cognitivas. 
Escritura académica: un modelo metodológico efectivo basado en tareas y enfoque cooperativo / Angie Neira Martínez y Anita Ferreira Cabrera

El aprendizaje cooperativo tiene como objetivo maximizar, a través de distintas técnicas, los beneficios de la cooperación entre los estudiantes (Jacobs y Small, 2003). También puede decirse que es percibido como aquellos procedimientos y técnicas pedagógicas que llevan al alumno a aprender conceptos y a desarrollarse socialmente (Ghaith, 2003). Lo anterior dependerá de la composición del grupo y de qué manera se organiza para llevar a cabo una tarea.

Es necesario, también, mencionar que el aprendizaje cooperativo va de la mano del enfoque por tareas. Esto debido a que el primero debe estar contextualizado en una situación comunicativa dada y guiado por tareas concretas y en las cuales el estudiante tenga un rol y una responsabilidad. En esto último se diferencia el aprendizaje cooperativo del trabajo en grupos.

\section{Diseño de la Investigación}

Este estudio tiene como objetivo principal proponer y aplicar un modelo metodológico que capacite a estudiantes universitarios en la producción de informes académicos estructural y lingüísticamente adecuados. Dicho objetivo surge frente a la hipótesis de que los estudiantes que sean partícipes de la metodología de enseñanza basada en tareas (grupo experimental) lograrán incrementar su aprendizaje de forma significativa acerca de cómo producir un informe académico, al compararlos con los obtenidos por aquéllos que estén expuestos a una metodología tradicional (grupo control).

La investigación está basada en un diseño experimental longitudinal pre-post test con grupo control, en el cual se compara el incremento en el aprendizaje sobre el discurso académico obtenido por dos grupos. La primera medición se realizó en una etapa inicial, anterior a la intervención pedagógica, en la que se les aplicó un pre test. La segunda se llevó a cabo al finalizar dicha intervención, en la que se les aplicó un post test.

\subsection{La Muestra}

La muestra se compone de estudiantes de una universidad tradicional de Concepción, Chile, divididos en dos grupos: uno experimental y otro de control. Cada uno de los grupos se encuentra compuesto por veintiún sujetos, en su totalidad de sexo masculino, de entre 19 y 23 años de edad y cursando el quinto semestre de su carrera. 


\subsection{Aplicación y Metodología}

El experimento se desarrolló a lo largo de ocho semanas, en las cuales ambos grupos tenían designadas tres horas de clase, correspondientes a asignaturas destinadas a mejorar sus competencias lingüísticas. Estas tres horas, para el grupo control, eran en su totalidad en aula. Para el grupo experimental, en cambio, sólo dos se realizaban en aula y la tercera se llevaba a cabo en un laboratorio de computación.

Para ambos grupos los tópicos que se trataron fueron los mismos, clasificados en los ítemes de uso de la lengua y estructura del informe académico. El primer ítem se limitó a los temas de conexión en el texto escrito y concordancias gramaticales. En el segundo se abordaron las etapas de la construcción de dicho género discursivo: organización (la investigación, la planificación), estructuración (basada en una organización tripartita de introducción, desarrollo/marco teórico, conclusión), escritura (inclusión de citas textuales y paráfrasis) e inclusión de referencias bibliográficas.

Cabe mencionar que en las semanas primera y octava ambos grupos fueron sometidos a evaluación. Durante la primera semana desarrollaron el pre test. Durante la octava realizaron el post test.

\subsubsection{Metodología Tradicional}

Se ha considerado como metodología tradicional a una práctica común en el desarrollo de clases de español como lengua materna en situaciones comunicativas académicas. Se trata, principalmente, de clases expositivas combinadas con la realización de actividades que se revisan en conjunto, ya sea en la pizarra o de manera oral. Éstas, además, tienen un desarrollo lineal. Esto es lo que Skehan (2008) llama las 3p: presentación, práctica y producción.

Las clases correspondientes a esta metodología se organizan en tres partes. En la primera, el profesor expone el tema a tratar; en la segunda se realiza una actividad de escritura relacionada con dicho tema y en la tercera se revisa en conjunto la actividad desarrollada. La exposición del profesor es apoyada por un apunte que se les entrega a los estudiantes o por una presentación con diapositivas proyectada desde el computador. Además de dar a conocer los temas y conceptos, éste va haciendo preguntas para verificar los conocimientos previos de los estudiantes y para cerciorarse de que han entendido los nuevos. Para aclarar lo que está exponiendo utiliza ejemplos y analogías. 
Escritura académica: un modelo metodológico efectivo basado en tareas y enfoque cooperativo / Angie Neira Martínez y Anita Ferreira Cabrera

Las actividades que se desarrollan en clase se entregan a los estudiantes en papel y se llevan a cabo individualmente. Una vez terminadas, son revisadas en conjunto, ya sea de manera oral o de forma escrita, es decir, se les pide a algunos alumnos que lean en voz alta el resultado de su ejercicio o que pasen a la pizarra y lo escriban. Esto dependerá de la naturaleza de la actividad.

En cuanto a la distribución de los tópicos, las primeras dos semanas (6 horas) están destinadas al tratamiento del ítem uso de la lengua y las cuatro restantes (12 horas), al de estructura del informe académico.

\subsubsection{Módulo de intervención lingüística}

Las tareas son el centro de este modelo metodológico, las que se desarrollan en conjunto con el trabajo cooperativo y con el uso de recursos computacionales. Además, se tratan de manera paralela los tópicos de estructura del informe académico y de formas lingüísticas. Cabe destacar que todos los temas seleccionados para diseñar los materiales de esta metodología se relacionan con el área de estudio de los alumnos (en este caso, informática).

Las clases son divididas de la siguiente forma:

Dos horas de clase en aula (o, dependiendo de la naturaleza de la tarea, en laboratorio de computación), en las cuales se abordan los temas referentes a la estructura del informe académico.

Una hora en laboratorio de computación, enfocada en el tratamiento de formas o estructuras lingüísticas.

Las clases cuyo eje es el desarrollo estructural y de contenido de un informe académico (o clases de contenido) son guiadas por el profesor y están organizadas en una secuencia compuesta por una pre tarea y una o más tareas de proceso. Además, los estudiantes se organizan en pares, los que trabajan en equipo con el fin de desarrollar un texto de forma cooperativa.

La pre tarea consiste en que cada par responde una serie de entre dos y seis preguntas referidas al tema que se abordará posteriormente en la tarea, cuyo objetivo es activar los conocimientos previos de los estudiantes. Una vez que terminan de contestar, se comentan las respuestas en conjunto de forma oral y se unifican los criterios y creencias de los alumnos. Después de ello, se les entrega una pauta de contenidos con la exposición del tema de forma breve y, a partir de ella, se indica la 
tarea que tendrán que llevar a cabo. Este apunte es leído por uno de los estudiantes en voz alta y el profesor explica aquello que no ha quedado claro. Al terminar la lectura, los pares desarrollan la tarea destinada para esa clase.

De esta forma, se construye, clase a clase y paso a paso, un informe académico de investigación bibliográfica en pares. Todas las tareas realizadas durante las clases en aula son archivadas en una carpeta. En el caso de esta investigación, este informe fue terminado, entregado y evaluado previo a la realización del post-test.

Las clases virtuales (que durante la investigación se llevan a cabo en un laboratorio de computación) se centran en el uso de formas lingüísticas, principalmente, en las concordancias gramaticales y la conexión en el texto escrito. Cada uno de estos temas fue dividido en dos módulos, esto es, se llevaron a cabo dos clases del primero y dos del segundo.

En suma, este modelo metodológico está centrado en el estudiante y en las tareas que éste lleva a cabo. El foco está en el desarrollo de sus habilidades a través de la práctica en clase.

\subsection{Los instrumentos de evaluación}

Para evaluar el incremento del aprendizaje de cada uno de los grupos se optó por aplicar un pre test antes de la intervención pedagógica y un post test al finalizar dicha intervención. Ambos fueron llevados a cabo a partir de un mismo instrumento de evaluación: un informe académico de investigación bibliográfica.

Para producir cada uno de los informes académicos solicitados (pre y post test) se les entregó a los estudiantes una pauta de entrega, la que indica los requisitos con los que debe cumplir el texto. En ella se plantean los requerimientos tanto de formato como de estructura. Además, se les propone un número limitado de temas, los cuales se relacionan con su área de estudio.

Para la evaluación del informe académico se utilizó una pauta de corrección. Ésta contiene los criterios de evaluación que se dividieron en dos ítems: el I, "estructura de un informe académico" y el II, "uso de la lengua”. El primer ítem comprende 32 de los 48 puntos de la evaluación, y el segundo ítem, los 16 restantes. Para calificar cada criterio se aplicaron los conceptos "cumplido" (equivalente a 2 puntos), "medianamente cumplido" (1 punto) y "no cumplido" (0 punto). 
Escritura académica: un modelo metodológico efectivo basado en tareas y enfoque cooperativo / Angie Neira Martínez y Anita Ferreira Cabrera

\section{Presentación y análisis de resultados}

Los datos obtenidos del pre y post test demuestran en gran medida el éxito de la investigación. Para comprobar su validez a nivel estadístico se aplicó la prueba ( o t de student) que indica si la diferencia al comparar dos medias es significativa. Esta fue aplicada con una confianza del 95\%.

Al comparar las medias obtenidas en pre y post test por cada uno de los grupos, se puede apreciar que ambos avanzaron en cuanto a sus puntajes, sin embargo, el grupo experimental ostenta una mayor diferencia que el grupo control. En el gráfico 1, se puede observar que la diferencia de los promedios entre los grupos en el pre test es mínima, de sólo un punto. En el post test, es posible apreciar una diferencia mayor, ya que el grupo experimental logró un promedio ocho puntos más alto que el del grupo control.

Gráfico 1: Comparación de los resultados obtenidos por el grupo experimental y el grupo control en pre y post test.

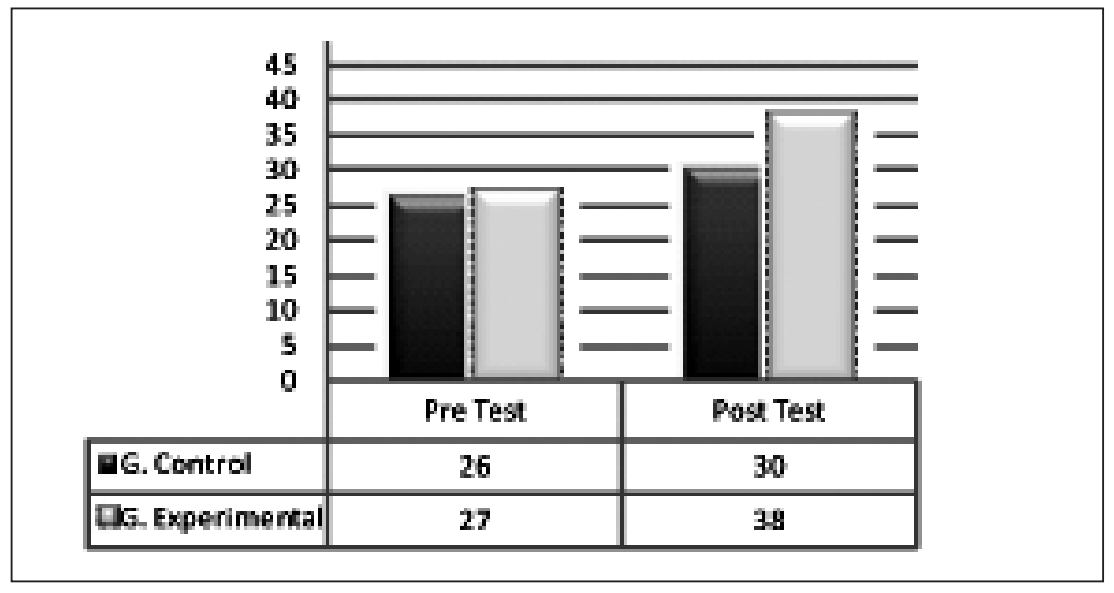

Lo anterior es corroborado por el análisis estadístico, ya que al comparar las medias de pre y post test del grupo control se puede observar un avance avalado por un valor $\mathrm{p}=0,01$, con un estadístico $t=-2,7$. En el grupo experimental se puede apreciar un avance más significativo, en un valor $\mathrm{p}=0,0000002$, con un estadístico $\mathrm{t}=-6,4$.

El ítem "estructura del informe académico" fue el que arrojó una mayor diferencia de resultados entre los grupos experimental y de control. A nivel estadístico, al comparar las diferencias de ambos grupos en este ítem (donde $\mathrm{Hl}=\mathrm{G}$. Control $<\mathrm{G}$. Experimental) el valor $\mathrm{p}=0,001$, con un estadístico $t=-3,5$. Esto evidencia que el grupo experimental obtuvo mejores resultados. 
Sin duda, en este ítem del instrumento de evaluación utilizado como pre y post test se comprueba la eficacia de la metodología basada en tareas que se sustenta, además, en el paradigma de la escritura como un proceso.

La diferencia en los resultados no solo se ve reflejada en la comparación de las medias obtenidas por cada grupo, sino por los rangos de puntajes en los que se ubican los estudiantes, representados en la tabla 1. En el grupo control solo un $10 \%$ de los estudiantes llegó al rango más alto de este ítem, entre 28 y 32 puntos, y ningún estudiante obtuvo el puntaje máximo. En el grupo experimental el 33\% se ubicó en ese rango, de los cuales un 10\% llegó al puntaje máximo. Asimismo, en el rango inmediatamente inferior, entre 24 y 27 puntos, solo el 23,5\% de los sujetos del grupo control se ubicó allí, mientras el 38\% del grupo control se encontraba en ese nivel. Por ende, es posible decir que el grupo experimental concentra la mayoría de sus integrantes en los dos intervalos más altos de puntaje, mientras el grupo control muestra una mayor dispersión de los sujetos en los distintos rangos de puntaje.

Tabla 1: Distribución de porcentajes de estudiantes del grupo experimental y del grupo control por rangos de puntajes obtenidos en pre y post test en el ítem "estructura del informe académico".

\begin{tabular}{|c|c|c|c|c|}
\hline \multirow{2}{*}{ Puntajes } & \multicolumn{4}{|c|}{$\%$ de estudiantes } \\
\cline { 2 - 5 } & \multicolumn{2}{|c|}{ Grupo Control } & Grupo experimental \\
\hline & Pre-test & Post-test & Pre-test & Post-test \\
\hline Entre 28 y 32 & 0 & $10 \%$ & 0 & $33 \%$ \\
\hline Entre 24 y 27 & 0 & $23,5 \%$ & $5 \%$ & $38 \%$ \\
\hline Entre 20 y 23 & $19 \%$ & $19 \%$ & $10 \%$ & $19 \%$ \\
\hline Entre 16 y 19 & $38 \%$ & $19 \%$ & $48 \%$ & $5 \%$ \\
\hline Entre 12 y 15 & $38 \%$ & $5 \%$ & $33 \%$ & 0 \\
\hline Menos de 12 & $5 \%$ & $23,5 \%$ & $5 \%$ & $5 \%$ \\
\hline
\end{tabular}

En el ítem "uso de la lengua", al comparar las medias, las diferencias entre ambos grupos fueron mínimas. Si bien el grupo experimental tuvo mejores resultados (con $\mathrm{Hl}=$ Pre test $<$ Post test; $\mathrm{p}=0,0002 ; \mathrm{t}=-4,3$ ) que el grupo control (con $\mathrm{Hl}=$ Pre test $<$ Post test; $\mathrm{p}=0,002 ; \mathrm{t}=-3,4$ ), éstos no son significativos al comparar las diferencias obtenidas por ambos grupos (con $\mathrm{Hl}=\mathrm{G}$. Control < G. Experimental; $\mathrm{p}=0,22 ; \mathrm{t}=-1,2$ ). Esto 
Escritura académica: un modelo metodológico efectivo basado en tareas y enfoque cooperativo / Angie Neira Martínez y Anita Ferreira Cabrera

indica que ambas metodologías son igualmente efectivas, es decir, que la realización de ejercicios en papel es tan eficaz como su desarrollo en un computador.

A pesar de lo anterior, no hay que perder de vista que hubo avances en esa área. Esto se ve reflejado en que un gran porcentaje de estudiantes del grupo experimental (71\%) obtuvo entre 12 y 16 puntos, el rango máximo en este ítem, y un 14\% obtuvo el puntaje máximo. En el grupo control solo un 43\% se ubicó en ese nivel de puntaje, mientras un 5\% obtuvo el puntaje máximo. Esto se puede observar en la tabla 2.

Además, es necesario agregar que, según lo que se observó cuando los estudiantes acudían a las clases virtuales en laboratorio, era motivante para ellos asistir a estas clases e incluso manifestaban que debería haber más ejercicios en laboratorio.

Tabla 2: Distribución de porcentajes de estudiantes del grupo experimental y del grupo control por rangos de puntajes obtenidos en pre y post test en el ítem "uso de la lengua".

\begin{tabular}{|l|c|c|c|c|}
\hline \multirow{2}{*}{ Puntajes } & \multicolumn{4}{|c|}{$\%$ de estudiantes } \\
\cline { 2 - 5 } & Grupo Control & Grupo experimental \\
\hline & Pre-test & Post-test & Pre-test & Post-test \\
\hline Entre 12 y 16 & $10 \%$ & $43 \%$ & $19 \%$ & $71 \%$ \\
\hline Entre 8 y 11 & $76 \%$ & $47 \%$ & $71 \%$ & $24 \%$ \\
\hline Entre 4 y 7 & $14 \%$ & $10 \%$ & $10 \%$ & $5 \%$ \\
\hline Entre 0 y 3 & 0 & 0 & 0 & 0 \\
\hline
\end{tabular}

\section{Conclusiones}

La alfabetización académica es una necesidad en nuestro sistema educativo universitario. Los estudiantes necesitan herramientas que les permitan enfrentar con éxito sus estudios, para lo cual la lectura y escritura de textos académicos y especializados es fundamental. Esto es precisamente el modelo metodológico planteado: una herramienta para el desarrollo de las habilidades escritas de los alumnos aplicadas a un género discursivo particular, el informe académico de investigación bibliográfica.

Los resultados obtenidos evidencian que el modelo metodológico es efectivo y que los objetivos se han cumplido en gran parte. El éxito de la experiencia reside, principalmente, en aquellas clases que se realizaron

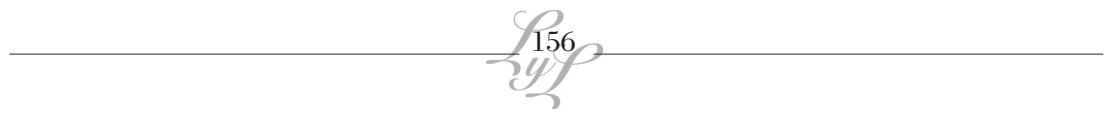


en aula, las cuales propiciaron una cooperación entre compañeros y una complementación entre ellos que les permitió desarrollar las habilidades y adquirir los conocimientos necesarios para enfrentar sin mayores dificultades la escritura de un informe académico de investigación bibliográfica. Este aprendizaje es transferible a la producción de otros tipos de informe, y, por qué no, a otros géneros académicos.

El modelo metodológico fue complementado con una perspectiva de género, esto es, se consideró como centro un género discursivo específico: el informe académico. Esta perspectiva permitió no solamente incrementar los aprendizajes de los estudiantes a nivel textual sino también a nivel discursivo. Los sujetos reconocieron la naturaleza socio-cultural del texto estudiado, los fines que guían su producción y la situación comunicativa en la que se inserta. Acompañado de aquello, comprendieron el registro que se debe utilizar en este género y su estructura global.

Uno de los principales componentes del diseño metodológico es el aprendizaje cooperativo. Éste se constituye en un gran aporte para el aprendizaje de los estudiantes. Al trabajar en pares, cada uno aprendió de los errores y aciertos del otro. El trabajo propició el diálogo y el intercambio de ideas, lo que incidió positivamente en cada uno de los alumnos del grupo experimental. Esto se vio reflejado no sólo en el informe académico que escribieron en conjunto, sino también en el que produjeron individualmente.

Otro componente fundamental para el éxito del modelo es el enfoque basado en tareas, que fue el núcleo metodológico del módulo de intervención lingüística. Éste proveyó a los estudiantes herramientas para analizar y producir paso a paso un informe académico de investigación bibliográfica, lo que les permitirá adaptarse con facilidad a otros géneros discursivos académicos. Por esta razón, es posible afirmar que los alumnos del grupo experimental recibieron no sólo las herramientas para producir un tipo de texto, sino que también adquirieron una base para desarrollar investigaciones futuras y producir géneros similares al aprendido, pero aún más especializados (como monografías, tesis, artículos especializados).

Si bien las tareas de apoyo lingüístico, que fueron realizadas en el laboratorio de computación, no arrojaron los resultados esperados, incrementaron la motivación de los estudiantes frente a la asignatura. Las falencias de este módulo pueden radicar en la cantidad de horas de clase que se destinaron para su desarrollo, la que fue muy inferior a las dedicadas a la estructura del informe académico. Aun así, el incremento del aprendizaje en este aspecto fue significativo. 
Escritura académica: un modelo metodológico efectivo basado en tareas y enfoque cooperativo / Angie Neira Martínez y Anita Ferreira Cabrera

Finalmente, el principal aporte de esta investigación radica en que provee un diseño metodológico efectivo para la enseñanza de la lengua materna en ambientes académicos. Además, ésta es una potencial fuente de debate y discusión en torno al tema de la alfabetización académica y a la necesidad de proponer metodologías contextualizadas y que consideren la naturaleza de la disciplina del estudiante.

\section{Bibliografía}

Bereiter, Carl y Marlene Scardamalia (1987). The Psychology of Written Composition. New Jersey: Lawrence Erlbaum Associates publishers.

Botta, Mirta (2001). Tesis, Monografías e Informes Buenos Aires: Biblos, 2002.

Chenowith, Ann y John Hayes (2001). "Fluency in Writing. Generating Text in L1 y L2". Written Communication 18(1): 80 - 98.

Ellis, Rod (2003). Task - based Language Learning and Teaching. Oxford, Inglaterra: Oxford University Press.

Estaire, Sheila (2004). La Enseñanza de Lenguas Mediante Tareas: Principios y Planificación de Unidades Didácticas. Madrid: Apuntes, MEELE, Universidad Antonio de Lebrija.

Figueras, Carolina y Marisa Santiago (2000). "Planificación". Estrella Montolío (coord.) Manual Práctico de Escritura Académica Vol. II. Barcelona: Ariel Lingüística.

Flower, Linda y John Hayes (1981). "A Cognitive Process Theory of Writing". College Composition and Communication, 32 (4): $365-387$.

Ghaith, Ghazi (2003). "Effects of the learning together model of cooperative learning on english as a foreign language reading achievement, academic self-esteem, and feelings of school alienation". Bilingual research journal, 27(3).

Grabe, William y Robert Kaplan (1996). Theory and Practice of Writing. Londres: Longman.

Harvey, Ana María y Daniel Muñoz (2006). "El género informe y sus representaciones en el discurso de los académicos". Estudios Filológicos, 41: 95-114.

Jacobs, George y John Small (2003). "Combining dictogloss and cooperative

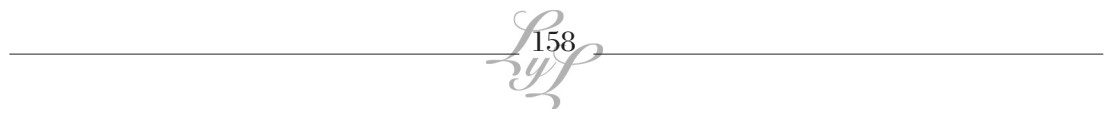


learning to promote language learning". The Reading matrix, 3 (1).

Marinkovich, Juana (2002). "Enfoques de proceso en la producción de textos escritos". Signos, 35(51-52): 217-230.

Nunan, David (1989). El Diseño de Tareas para la Clase Comunicativa. Madrid: Cambridge University Press.

(2001). Aspects of Task-Based Syllabus Design. The English Centre, University of Hong Kong.

Skehan, Peter (2008). A Cognitive Approach to Language Learning. Oxford University Press.

Tapia, Mónica, Gina Burdiles y Beatriz Arancibia (2003). "Aplicación de una pauta diseñada para evaluar informes académicos universitarios". Signos, 36(54): 249-257.

Trujillo, Fernando (2002). "Aprendizaje Cooperativo para la Enseñanza de la Lengua". Publicaciones de la Facultad de Educación y Humanidades - Campus de Melilla, Universidad de Granada, 32: 147-162. 